\title{
GNAS1 T393C Polymorphism and Disease Progression in Patients with Malignant Melanoma
}

\author{
U. H. Frey ${ }^{1}$ 2*, A. Fritz ${ }^{3 *}$, S. Rotterdam ${ }^{3}$, K. W. Schmid ${ }^{4}$, A. Potthoff ${ }^{3}$, P. Altmeyer ${ }^{3}$, W. Siffert ${ }^{1}$, \\ N. H. Brockmeyer ${ }^{3}$ \\ ${ }^{1}$ Institut für Pharmakogenetik, Universität Duisburg-Essen, Universitätsklinikum Essen, Essen ,Germany, \\ ${ }^{2}$ Institut für Anästhesiologie und Intensivmedizin, Universität Duisburg-Essen, Universitätsklinikum Essen, Essen ,Germany, \\ ${ }^{3}$ Klinik für Dermatologie und Allergologie, St. Josef Hospital, Bochum, Germany, \\ ${ }^{4}$ Institut für Pathologie und Neuropathologie, Universität Duisburg-Essen, Universitätsklinikum Essen, Essen, Germany
}

\begin{abstract}
Background: Once metastasized, despite a variety of therapeutic options, the prognosis of patients with malignant melanoma (MM) is still poor. Therefore, the search for reliable markers to identify patients with high risk of disease progression is of high clinical importance. We have recently shown that TT genotypes of the single-nucleotide polymorphism (SNP) T393C in the gene GNAS1 are significantly associated with better outcome in a variety of carcinomas.

Patients: In the present study we assessed whether the T393C SNP is also related to the clinical course in MM. 328 patients with MM were retrospectively genotyped and genotypes were correlated with clinical outcome.

Results: While the allele frequency in the MM group (fC 0.52) did not significantly differ from that of healthy blood donors, the T393C SNP was associated with tumor progression of MM. Carriers of the C-allele showed a significantly more severe tumor progression as estimated from the time period to develop metastasis (HR 2.2, 95\% CI 1.1-3.2, $\mathrm{p}=0.017$ ). Proportions of 5-year metastasis-free intervals were $87.1 \%$ for TT genotypes and $66.0 \%$ for C-allele carriers. Moreover, multivariable Cox regression analysis including tumor stage and melanoma subtype proved the T393C polymorphism to be an independent factor for metastasis $(\mathrm{p}=0.012)$.

Conclusions: In summary, the GNAS1 T393C SNP represents a genetic host factor for predicting tumor progression also in patients with MM; genotyping of this SNP may contribute to better define patients who could benefit from an early individualized therapy.
\end{abstract}

Key words: polymorphism, G Protein, melanoma, prognostic factor

\section{INTRODUCTION}

Early detection of metastasis is one of the aims of follow-up examination in patients with malignant melanoma since once metastasized, despite a variety of

*These authors contributed equally to this work. therapeutic options e. g. chemo- and/ or immunotherapy, patients suffering from malignant melanoma show an unfavorable prognosis. Therefore, it would be most desirable to identify patients with high risk of disease progression as early as possible [1]. Since laboratory tests are easy to handle and modestly expensive, a variety of different serum factors has been investigated for their potential relevance as prognostic markers in malignant melanoma [2-4]. To date, none of these markers is routinely used in the clinical setting. The most promising one at present, $\mathrm{S} 100$-protein, has been demonstrated in several studies to be a valid and reliable predictive marker for clinical progression and response to therapy in melanoma patients with advanced stages of disease $[5,6]$. However, a major disadvantage of S100-protein is its poor prognostic impact in earlystage melanoma patients [6-8].

The identification of new prognostic markers may help to better distinguish low from high risk disease apart from the classical histopathologic and clinical criteria and may unravel new targets for therapy. The mechanism of malignant transformation of melanocytes is poorly understood, but growth factors and their receptors play an important role in the progression of melanoma [9]. Moreover, alterations in the mitogen-activated protein kinase pathway are almost uniformly present in melanoma. While RAS itself is only infrequently mutated in melanoma [10] the downstream BRAF gene of the mitogen activated protein kinase pathway is mutated in $60 \%$ to $70 \%$ of superficial spreading melanomas [11]. On the other hand, a genetic host factor may offer a better stratification of patients into more refined risk categories without the need for tumor tissue. For example, the A61G single nucleotide polymorphism (SNP) in the EGF gene which operates via RAS and BRAF in melanocytic tumors has recently been associated with risk for metastasis in melanoma patients [12].

We have recently shown that the T393C polymorphism in the gene GNAS1, which encodes the G_s subunit of heterotrimeric $G$ protein, is significantly associated with the clinical outcome of patients suffering from bladder cancer [13], colorectal cancer [14], chronic lymphocytic leukaemia [15], and renal cell carcinoma [16]. We also demonstrated that the $\mathrm{T}$ allele of 
GNAS1 T393C polymorphism is associated with increased G $\alpha$ s mRNA expression in a variety of tissues, including tumor tissue [14]. In vitro experiments suggest that an increased expression of Gas is associated with enhanced apoptosis and that the second messenger cAMP, which functions downstream of $G$ proteins, plays a major role in this pro-apoptotic process [17-20]. Hence, an increased Gos expression with concomitantly enhanced apoptosis may be also associated with better survival in melanoma patients with T'T genotypes.

\section{Materials AND Methods}

\section{Patients}

This study comprises a total of 328 consecutive malignant melanoma patients (with a mean age of $54.2 \pm$ 16.4 years) treated at the Department of Dermatology and Allergology, Ruhr-University Bochum (Bochum, Germany) between 1995 and 2000 with disease progression being continuously documented. The clinical staging was performed according to the American Joint Committee on Cancer (AJCC 1997). Briefly, stages I and II included patients with primary melanoma, stage III included patients with regional lymph node and/or in-transit metastases and stage IV included patients with distant metastases. Patients were treated according to therapy protocols of the Dermatologic Cooperative Oncology Group (DeCOG) including cytostatic drugs (dacarbacine, cisplatinum, temozolomide, vincristine) and immunomodulatory agents [interferon(IFN)] in different combinations and schedules. Follow-up was performed in at least 3-months intervals including physical examination, $\mathrm{x}$-ray of the chest, ultra- sound of the abdomen and lymph nodes as well as blood chemistry. Patients in advanced disease stages additionally underwent computer tomography of the brain and szintigraphy of the skeleton. Detailed clinical characteristics of the study patients are summarized in Table 1 . The retrospective analysis and genotyping of DNA from paraffin-embedded tissues in an anonymous form was approved by the ethical committee of the University Hospital Essen (Essen, Germany).

\section{GENOTYPING}

DNA was extracted from paraffin-embedded tissue samples using a commercially available kit (QIAAmp, Qiagen, Hilden, Germany). Genotypes of the T393C

Table 1.

\begin{tabular}{|c|c|c|c|c|c|}
\hline & All & TT & $\mathrm{TC}$ & CC & $\mathrm{P}$ \\
\hline $\mathrm{N}(\%)$ & 328 & $69(21.0)$ & $149(45.4)$ & $110(33.5)$ & \\
\hline Gender (m/f) & $167 / 161$ & $30 / 39$ & $86 / 63$ & $51 / 59$ & 0.074 \\
\hline Mean age at diagnosis & 54.2 & 55.0 & 54.4 & 53.4 & 0.517 \\
\hline $\begin{array}{l}\text { UICC Category. } \\
\text { I }\end{array}$ & 202 (61.6) & $45(22.3)$ & $85(42.1)$ & $72(35.6)$ & UICC Category. \\
\hline II & $72(22.0)$ & $13(18.1)$ & $34(47.2)$ & $25(34.7)$ & \\
\hline III & $47(14.3)$ & $9(19.1)$ & $30(63.8)$ & $8(17.0)$ & \\
\hline IV & $7(2.1)$ & $2(28.6)$ & $0(0)$ & $5(71.4)$ & 0.683 \\
\hline \multicolumn{6}{|l|}{ Type } \\
\hline SSM & 153 & 31 & 71 & 51 & \\
\hline NM & 38 & 8 & 16 & 14 & \\
\hline LMM & 34 & 9 & 14 & 11 & \\
\hline ALM & 30 & 7 & 16 & 7 & \\
\hline UCM & 73 & 14 & 32 & 27 & \\
\hline \multicolumn{6}{|l|}{ Tumor thickness (mm) } \\
\hline$<0,75$ & $191(58.2)$ & $40(20.9)$ & $80(41.9)$ & $71(37.2)$ & \\
\hline $0,75-1,5$ & $55(16.8)$ & $14(25.5)$ & $27(49.1)$ & $14(25.5)$ & \\
\hline $1,5-4$ & $58(17.7)$ & $8(13.8)$ & $31(53.4)$ & $19(32.8)$ & \\
\hline$>4$ & $24(7.3)$ & $7(29.2)$ & $11(45.8)$ & $6(25.0)$ & 0.385 \\
\hline \multicolumn{6}{|l|}{ Clark level } \\
\hline I & $51(15.5)$ & $10(19.6)$ & $22(43.1)$ & $19(37.3)$ & \\
\hline II & $40(12.2)$ & $10(25.0)$ & $15(37.5)$ & $15(37.5)$ & \\
\hline III & $114(34.8)$ & $24(21.1)$ & $50(43.9)$ & $40(35.1)$ & \\
\hline IV & $92(28.0)$ & $20(21.7)$ & $45(48.9)$ & $27(29.1)$ & \\
\hline V & $10(3.0)$ & $2(20.0)$ & $6(60.0)$ & $2(20.0)$ & 0.333 \\
\hline \multicolumn{6}{|l|}{ Node } \\
\hline 0 & $305(93.0)$ & $66(21.6)$ & $135(44.3)$ & $104(34.1)$ & \\
\hline 1 & $13(4.0)$ & $3(23.1)$ & $6(46.2)$ & $4(30.8)$ & \\
\hline 2 & $10(3.0)$ & $0(0)$ & $8(80.0)$ & $2(20.0)$ & 0.865 \\
\hline \multicolumn{6}{|l|}{ Distant metastasis } \\
\hline No & $321(97.9)$ & $67(20.9)$ & $149(46.4)$ & $105(32.7)$ & \\
\hline Yes & $7(2.1)$ & $2(28.6)$ & $0(0,0)$ & $5(71.4)$ & 0.266 \\
\hline
\end{tabular}

M; male; f, female; SSM, superficial spreading melanoma; NM, nodular melanoma; LMM, lentigo maligna melanoma; ALM, acral lentiginous melanoma; UCM, unclassified melanoma 
polymorphism were determined by PCR using the following primers: forward primer 5'-CTCCTAACTGA CATGGTGCAA-3' and reverse primer 5'-TAAGGC CACACAAGTCGGGGT-3'. After denaturation at $94^{\circ} \mathrm{C}, 35$ cycles of DNA amplification were performed using Taq PCR Mastermix (Eppendorf, Hamburg, Germany) at $94^{\circ}$ for $45 \mathrm{sec}, 58^{\circ} \mathrm{C}$ for $40 \mathrm{sec}-$ onds, and $72^{\circ}$ for 45 seconds. The 345 bp PCR products were digested using the restriction enzyme FokI and analyzed on a $2 \%$ agarose gel. The unrestricted products (345 bp) represented the TT genotype; the completely restricted products (259 and $86 \mathrm{bp}$ ) represented the CC genotype.

\section{STATISTICAL ANALYSis}

The clinical outcome analyzed in this study was survival, and metastasis dependent on UICC stages, melanoma subtypes, and T393C genotypes. KaplanMeier plots and the log-rank test were used to evaluate the relationship between UICC stages, melanoma subtypes or T393C genotypes, and clinical outcome from the date of the primary diagnosis to the end of follow-up. The effects of gender, age at diagnosis, UICC stages, and T393C genotypes as prognostic factors for the clinical outcome were analyzed by stepwise multivariate Cox regression analysis. Hazard ratios and 95\% confidence intervals $(95 \% \mathrm{CI})$ were calculated from the Cox regression model including all factors for multivariate analysis and for the indicated factor for univariate analysis. Contingency tables and the Pearson's chi-square test were used for categorical variables using T393C genotypes. Linear ANOVA was used for comparison of continuous variables where appropriate. Differences were regarded significant at $\mathrm{P}<0.05$. All statistical analyses were done using SPSS 13.0 (SPSS, Chicago, IL) or GraphPad Prism 4.0 (GraphPad Software, San Diego, CA). Continuous variables are given as means $\pm \mathrm{SD}$ or $\pm \mathrm{SE}$ as indicated.

\section{RESULTS}

\section{Genotype Distribution}

Demographic characteristics and tumor stage in the whole patient group and by T393C genotypes are displayed in Table 1 . The frequency of the $C$ allele in the patient group was 0.52 and genotype distribution was compatible with the Hardy-Weinberg equilibrium. Genotypes and allele frequency of patients were comparable with those of healthy white blood donors; details of this control group have been published previously [21]. This argues against an association of T393C genotypes with an increased susceptibility for malignant melanoma. We found no genotype association with age at time of first diagnosis. Moreover, genotypes were not significantly associated with tumor localization, UICC classification, or tumor thickness (Table 1).

\section{T393C Genotype And Clinical Follow up}

During follow up, 85 patients $(25.9 \%)$ experienced metastases and $88(28.1 \%)$ died. In order to confirm that our sample was representative for patients with malignant melanoma we calculated Kaplan-Meier curves for overall survival depending on the UICC stages and tumor subtype. As shown in Fig. 1 A-B, overall survival was significantly dependent upon AJCC stage and tumor type. Five-year survival rates were $90.4 \%$ for UICC I, $77.1 \%$ for UICC II, $52.4 \%$ for UICC III, and $28.6 \%$ for UICC IV. Regarding tumor types, 5-year survival rates were $83.4 \%$ for SSM, $73.3 \%$ for NM, $87.3 \%$ for LMM, 75.0\% for ALM and $79.4 \%$ for UCM. These findings are comparable with those from the literature [22].

Concerning genotypes of the T393C polymorphism we observed no association with overall survival. However, we detected a significant allele-dependent influence upon tumor progression. GNAS1 393C allele carriers displayed a higher risk for metastasis ( $\mathrm{C}$ versus TT: $2.2,95 \%$ CI 1.1-3.2, $\mathrm{p}=0.017)$ compared to T393 homozygous patients. Proportions of 5-year metastasis free intervals were $87.1 \%$ for TT genotypes and $66.0 \%$ for $\mathrm{C}$-allele carriers, respectively (Fig. 2).

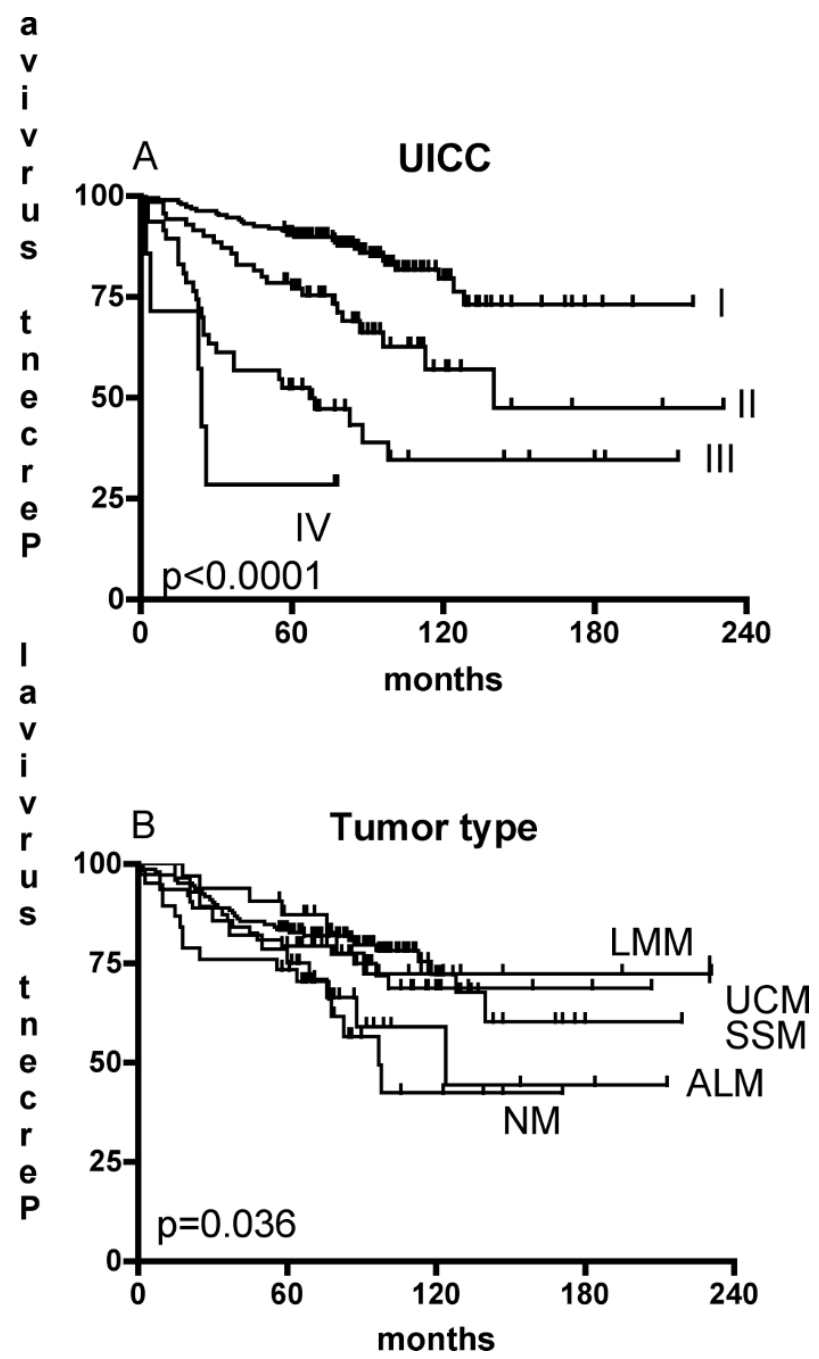

Fig, 1. Kaplan-Meier curves for overall survival in 328 patients with malignant melanoma based on the UICC stage (A) and tumor subtype (B). SSM, superficial spreading melanoma; NM, nodular melanoma; LMM, lentigo maligna melanoma; ALM, acral lentiginous melanoma; UCM, unclassified melanoma. 


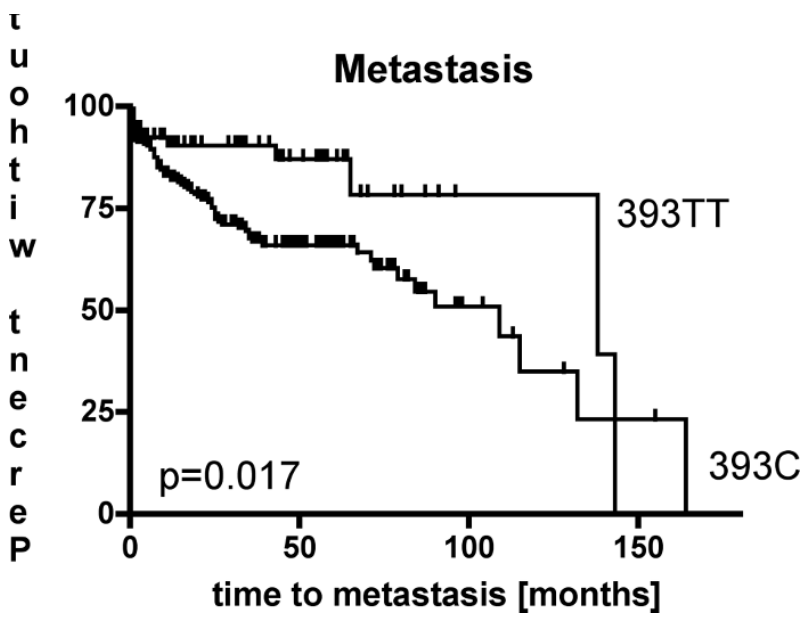

Fig. 2. Kaplan-Meier curves for metastasis-free survival in 328 patients with malignant melanoma based on T393C alleles.

Table 2. Multivariable Cox proportional hazard model for metastasis in 328 patients with malignant melanoma.

\begin{tabular}{llll}
\hline & \multicolumn{3}{c}{ Metastasis } \\
\cline { 2 - 4 } & Hazard ratio & $95 \%$ CI & $\mathrm{p}$ \\
\hline $\begin{array}{c}\text { Age at diagnosis } \\
\text { Gender } \\
\text { Male }\end{array}$ & 1.0 & $0.98-1.02$ & 0.802 \\
Female & $1 *$ & & \\
UICC Stage & 0.96 & $0.61-1.53$ & 0.866 \\
I & $1 *$ & & \\
II & 1.91 & $0.96-3.81$ & 0.067 \\
III & 12.19 & $6.30-23.56$ & $<0.001$ \\
IV & 21.47 & $6.03-76.49$ & $<0.001$ \\
MM Type & $1 *$ & & \\
SSM & 1.89 & $0.92-3.88$ & 0.081 \\
NM & 2.75 & $1.14-6.2$ & 0.024 \\
LMM & 1.56 & $0.74-3.33$ & 0.239 \\
ALM & 2.38 & $1.23-4.58$ & 0.010 \\
UCM & & & \\
T393C & $1 *$ & $1.22-5.32$ & 0.012 \\
TT & 2.55 & & \\
C & & &
\end{tabular}

*reference group. CI, confidence interval. SSM were chosen as reference because they represent the largest subgroup of melanomas. SSM, superficial spreading melanoma; NM, nodular melanoma; LMM, lentigo maligna melanoma; ALM, acral lentiginous melanoma; UCM, unclassified melanoma

In the multivariable analysis with gender, age at diagnosis, tumor stage, and melanoma subtype, the T393C polymorphism was an independent marker for tumor progression. The hazard ratio for patients with C-alleles was 2.55 for metastasis compared to homozygous 393T allele carriers (Table 2).

\section{Discussion}

Although UICC stages generally correlate with outcome in a large percentage of cancer patients, refined prediction of the individual disease course would facilitate clinical decision making, e.g. early requirement of adjuvant therapy. The familial melanoma syndromes are associated with germ line mutations in three highly penetrant gene products: p16, alternate reading frame, and cyclin-dependent kinase 4. Certain variants in a low-penetrance gene, MC1R, the melanocortin 1 receptor gene, increase melanoma risk to a lesser extent and act as genetic modifiers when cosegregating with a deleterious p16 gene [23]. Since the immune response against malignant melanoma cells can be influenced by cytokines with potentially inhibitory effects on tumor cell growth, SNPs in cytokine genes associated with reduced cytokine production are expected to influence susceptibility to cancer, including malignant melanoma. Genotypes associated with reduced production of pro-inflammatory and immunomodulatory TNF-alpha, IFN-gamma, and IL-6, and anti-inflammatory IL10 and TGF-beta1 could be involved in mechanisms of cancer progression and escape from immunosurveillance [24]. Genotypic variations in the IL10 promotor are significantly associated with an increased risk for malignant melanoma and may be associated with a poor outcome [25]. One of the aims of the present study was, therefore, to investigate whether the common T393C SNP in the gene GNAS1, may be predictive for survival or disease-free survival in patients with malignant melanoma.

The mean age of the patients of the present malignant melanoma series was 54.2 years and the median follow-up time was 80 months. Distribution of tumor stage and subtype of the whole series were compatible with data reported in the literature. Results of the German Central Register of Malignant Melanoma showed a mean age of 58.1 year and a similar distribution of subtypes as reported here [26]. Beside the role of tumor stage and tumor thickness as the most important prognostic factor in patients with malignant melanoma, our data suggest a potential predictive use of the GNAS1 T393C SNP as an independent prognostic factor in malignant melanoma. C-allele carriers have an increased risk for tumor progression when compared to patients with the homozygous 393TT genotype, the latter group showing both a longer relapse-free survival and a later requirement for secondary therapy. Together with our previous observations showing that the T393C SNP is a predictive marker for outcome in bladder cancer [13], renal cell carcinoma [16] and colorectal cancer [14], the present results confirm the potential role of this SNP as a universal genetic marker for predicting tumor progression. The GNAS1 TT genotype is associated with increased Gas mRNA expression in different tissues14 which led us to hypothesize that the T393C exchange itself could have an effect upon mRNA stability. Interestingly, determinants of mRNA stability have been described in the coding region of some other genes [27-29]. Different in vitro experiments suggest that increased expression of Gas is associated with enhanced apoptosis [17-19]. The second messenger cyclic AMP, which is generated subsequently to activation of Gas, seems to play a major role in this proapoptotic process. Cyclic AMP can augment or suppress ERK activity, depending on the cell type. As an example, CRAF is inhibited by protein kinase A, which is one of the downstream effectors of cAMP 
[30]. Cyclic AMP is produced in response to melanocytic agonists such as $\alpha$-melanocyte-stimulating hormone acting through the melanocortin 1 receptor [31] resulting in activation of Gas. Although this is only able to weakly stimulate proliferation, cAMP is closely associated with melanocyte differentiation (e. g. stimulation of melanin synthesis) [32].

A recent report has shown that cAMP blocks CRAF activity in melanocytes resulting in suppression of the oncogenic activity of CRAF in these cells [33]. This process could be of particular importance in TT genotypes in which Gas expression is increased. However, CRAF is not required for MEK/ERK signalling in melanoma cells when BRAF is mutated [34, 35]. Late mutations in the BRAF gene could, therefore, mitigate the T393C genotype effect which may explain the lack of genotype-dependent differences in overall survival. This hypothesis is supported by observations from studies in different types of cancer in which BRAF mutations are rarely events and in which then the T393C SNP affects overall survival [36]. The goal of ongoing studies is to unravel both the detailed molecular mechanisms by which the silent C393T SNP contributes to differential Gas expression in human tissues and how this may change the phenotype of different tumor cells.

The authors state no conflict of interest.

\section{REFERENCES}

1. Rebmann V, Ugurel S, Tilgen W et al. Soluble HLA-DR is a potent predictive indicator of disease progression in serum from early-stage melanoma patients. Int J Cancer. 2002; 100: 580-5.

2. Bosserhoff AK, Kaufmann M, Kaluza B et al. Melanomainhibiting activity, a novel serum marker for progression of malignant melanoma. Cancer Res. 1997; 57: 3149-53.

3. Ugurel S, Rappl G, Tilgen W et al. Increased serum concentration of angiogenic factors in malignant melanoma patients correlates with tumor progression and survival. J Clin Oncol. 2001; 19: 577-83.

4. Ugurel S, Rappl G, Tilgen W et al. Increased soluble CD95 (sFas/CD95) serum level correlates with poor prognosis in melanoma patients. Clin Cancer Res. 2001; 7: 1282-6.

5. Hauschild A, Engel G, Brenner W et al. Predictive value of serum S100B for monitoring patients with metastatic melanoma during chemotherapy and/or immunotherapy. Br J Dermatol. 1999; 140: 1065-71.

6. Hauschild A, Engel G, Brenner W et al. S100B protein detection in serum is a significant prognostic factor in metastatic melanoma. Oncology. 1999; 56: 338-44.

7. Guo HB, Stoffel-Wagner B, Bierwirth T et al. Clinical significance of serum S100 in metastatic malignant melanoma. Eur J Cancer. 1995; 31A: 924-8.

8. Henze G, Dummer R, Joller-Jemelka HI et al. Serum S100--a marker for disease monitoring in metastatic melanoma. Dermatology. 1997; 194: 208-12.

9. Streit S, Mestel DS, Schmidt M et al. FGFR4 Arg388 allele correlates with tumour thickness and FGFR4 protein expression with survival of melanoma patients. Br J Cancer. 2006; 94: 1879-86.

10. Tsao H, Zhang X, Fowlkes K et al. Relative reciprocity of NRAS and PTEN/MMAC1 alterations in cutaneous melanoma cell lines. Cancer Res. 2000; 60: 1800-4.

11. Davies H, Bignell GR, Cox C et al. Mutations of the BRAF gene in human cancer. Nature. 2002; 417: 949-54.
12. Okamoto I, Roka F, Krogler J et al. The EGF A61G polymorphism is associated with disease-free period and survival in malignant melanoma. J Invest Dermatol. 2006; 126: $2242-6$.

13. Frey UH, Eisenhardt A, Lummen G et al. The T393C polymorphism of the G alpha s gene (GNAS1) is a novel prognostic marker in bladder cancer. Cancer Epidemiol Biomarkers Prev. 2005; 14: 871-7.

14. Frey UH, Alakus H, Wohlschlaeger J et al. GNAS1 T393C polymorphism and survival in patients with sporadic colorectal cancer. Clin Cancer Res. 2005; 11: 5071-7.

15. Frey UH, Nuckel H, Sellmann L et al. The GNAS1 T393C Polymorphism Is Associated with Disease Progression and Survival in Chronic Lymphocytic Leukemia. Clin Cancer Res. 2006; 12: 5686-92.

16. Frey UH, Lummen G, Jager T et al. The GNAS1 T393C polymorphism predicts survival in patients with clear cell renal cell carcinoma. Clin Cancer Res. 2006; 12: 759-63.

17. Daaka Y, Luttrell LM, Lefkowitz RJ. Switching of the coupling of the beta2-adrenergic receptor to different $G$ proteins by protein kinase A. Nature. 1997; 390: 88-91.

18. Krumins AM, Barber R. Examination of the effects of increasing Gs protein on beta2-adrenergic receptor, Gs, and adenylyl cyclase interactions. Biochem Pharmacol. 1997; 54: 61-72.

19. Yang X, Lee FY, Sr., Wand GS. Increased expression of Gs(alpha) enhances activation of the adenylyl cyclase signal transduction cascade. Mol Endocrinol. 1997;11: 1053-61.

20. Yang W, White B, Spicer EK et al. Complex haplotype structure of the human GNAS gene identifies a recombination hotspot centred on a single nucleotide polymorphism widely used in association studies. Pharmacogenetics. 2004; 14: 741-7.

21. Siffert W, Forster P, Jockel KH et al. Worldwide ethnic distribution of the $G$ protein beta3 subunit $825 \mathrm{~T}$ allele and its association with obesity in Caucasian, Chinese, and Black African individuals. J Am Soc Nephrol. 1999; 10: 1921-30.

22. Balch CM, Buzaid AC, Soong SJ et al. Final version of the American Joint Committee on Cancer staging system for cutaneous melanoma. J Clin Oncol. 2001; 19: 3635-48.

23. Pho L, Grossman D, Leachman SA. Melanoma genetics: a review of genetic factors and clinical phenotypes in $\mathrm{fa}$ milial melanoma. Curr Opin Oncol. 2006; 18: 173-9.

24. Nikolova PN, Pawelec GP, Mihailova SM et al. Association of cytokine gene polymorphisms with malignant melanoma in Caucasian population. Cancer Immunol Immunother. 2007; 56: 371-9.

25. Martinez-Escribano JA, Moya-Quiles MR, Muro M et al. Interleukin-10, interleukin-6 and interferon-gamma gene polymorphisms in melanoma patients. Melanoma Res. 2002; 12: 465-9.

26. Buettner PG, Leiter U, Eigentler TK et al. Development of prognostic factors and survival in cutaneous melanoma over 25 years: An analysis of the Central Malignant Melanoma Registry of the German Dermatological Society. Cancer. 2005; 103: 616-24.

27. Duan J, Wainwright MS, Comeron JM et al. Synonymous mutations in the human dopamine receptor D2 (DRD2) affect mRNA stability and synthesis of the receptor. Hum Mol Genet. 2003; 12: 205-16.

28. Capon F, Allen MH, Ameen M et al. A synonymous SNP of the corneodesmosin gene leads to increased mRNA stability and demonstrates association with psoriasis across diverse ethnic groups. Hum Mol Genet. 2004; 13: 2361-8.

29. Tierney MJ, Medcalf RL. Plasminogen activator inhibitor type 2 contains mRNA instability elements within exon 4 of the coding region. Sequence homology to coding region instability determinants in other mRNAs. J Biol Chem. 2001; 276: 13675-84. 
30. Dumaz N, Light Y, Marais R. Cyclic AMP blocks cell growth through Raf-1-dependent and Raf-1-independent mechanisms. Mol Cell Biol. 2002; 22: 3717-28.

31. Kadekaro AL, Kanto H, Kavanagh R et al. Significance of the melanocortin 1 receptor in regulating human melanocyte pigmentation, proliferation, and survival. Ann N Y Acad Sci. 2003; 994: 359-65.

32. Hunt G, Todd C, Cresswell JE et al. Alpha-melanocyte stimulating hormone and its analogue Nle4DPhe7 alphaMSH affect morphology, tyrosinase activity and melanogenesis in cultured human melanocytes. J Cell Sci. 1994; 107 ( Pt 1): 205-11.

33. Dumaz N, Hayward R, Martin J et al. In Melanoma, RAS Mutations Are Accompanied by Switching Signaling from BRAF to CRAF and Disrupted Cyclic AMP Signaling. Cancer Res. 2006; 66: 9483-91.

34. Karasarides M, Chiloeches A, Hayward R et al. B-RAF is a therapeutic target in melanoma. Oncogene. 2004; 23: 6292-8.
35. Hingorani SR, Jacobetz MA, Robertson GP et al. Suppression of BRAF(V599E) in human melanoma abrogates transformation. Cancer Res. 2003; 63: 5198-202.

36. Davies H, Bignell GR, Cox C et al. Mutations of the BRAF gene in human cancer. Nature. 2002; 417: 949-54.

Received: April 9, 2010 / Accepted: May 3, 2010

Address for correspondence:

Prof. Dr. N. H. Brockmeyer

Klinik für Dermatologie und Allergologie

St. Josef Hospital

Gudrundstr. 56

44791 Bochum

Germany

Phone: +49-234-5093470

E-mail: n.brockmeyer@derma.de 